\title{
Study of a Multi-layers Quality of Service Model for ADHOC networks
}

\author{
Lakrami Fatima \\ STIC Laboratory Chouaib Doukkali University \\ Eljadida,MOROCCO
}

\author{
Elkamoun Najib \\ STIC Laboratory Chouaib Doukkali University \\ Eljadida,MOROCCO
}

\begin{abstract}
The quality of service support in MANETs has become an important requirement. However, the QOS support in a wireless networks is unlike that of the wireline's because wireless bandwidth is shared among neighboring nodes and the network topology continuously changes with node mobility. This condition requires communicating nodes to collaborate imperatively, in order to determinate the routes and to preserve the vital resources necessary to provide the QOS. We investigate in this paper an example of multi-layers quality of service management in MANETs, involving the following quality of services mechanisms: QOS routing with an OLSR extension with QOS support, EDCAF (Enhanced Distributed Coordinate Function) at MAC layer , and an hybrid FEC/ARQ scheme at link layer. We study the possibility of incorporating all of those protocols, as part of a collaborative independent logic, to provide a quality of service level better than that offered individually by each protocol. We use the principle of "complementarity of roles", which aims to enhance the transmission of sensitive traffic, without having to invoke any inter-layers exchange. We demonstrate through simulations that even this virtual cooperation improves different performance metrics (delay,loss rate...), on condition to select intelligently the principal actors .
\end{abstract}

\section{General Terms}

Quality of service, Mobile AD-hoc networks, multi-layers adaptation

\section{Keywords}

Wireless networks, multi-layers adaptation, OLSR, EDCAF, FEC.

\section{INTRODUCTION}

The ad hoc wireless networks consist of various computer systems, more or less complex, subsequently called nodes, having the ability to communicate independently by radio waves. The nodes can interact and cooperate to share services. A node can both communicate directly with others and serve as relay device. It will allow nodes presently out of radio range of each other to communicate. These networks are called adhoc to the extent that they do not require fixed infrastructure. They can exist temporarily to meet a specific need of communication. As an autonomous system, MANET should provide its own routing protocols and network management mechanisms. As a multi-hop wireless extension, it should provide a flexible and seamless communication among the users or access to the Internet. The design of communication protocols in wireless networks was initially destined to carry best effort traffic . The problem of the quality of service become more persistent due to increasing popularity of multimedia applications and pending commercial deployment of MANETs. Accordingly, the necessity to establish appropriate QOS mechanisms has became one of the most treated problems by the wireless network research community. In this context two solutions were proposed : the first one was to develop squarely new protocols with QOS basic support. The second one was to adapt the auxiliary functioning of existent communication protocols, to respond to QOS requirements, in order to preserve the compatibility and the serenity of the proposed QOS solutions.

This paper outlines the possibility of deploying a multi-layers QOS scheme, with the use of a series of protocols, operating at different network layers, to provide independently, a certain quality of service level. The core idea behind our approach is based on "the complementarity of roles", where the offered service of one protocol aims to fulfill the graps of other services offered by the rest of participating protocols. We propose an example of the usage of three communications protocols, widely treated by the literature: an extension of OLSR routing protocol with QOS support, EDCA an access method based on DCF with service differentiation; and at link layer we propose to use an hybrid FEC/ARQ mechanism in order to enhance the delay of the transmission of critical applications with a bandwidth optimization.

The remainder of this paper is organized as follow :section II describes related works, section III presents a review of the adaptation process, section VI describes our approach with a brief presentation of different deployed protocols, section $\mathrm{V}$ presents the simulation and the results while section IV concludes the paper .

\section{RELATED WORKS}

Compared with approaches based on cross-layers mechanism, the use of a multi-layers QOS ,without involving the interlayer exchanges, is not frequently treated .The idea was notably presented in [1], where authors propose a combination of IP and MAC layers. This work was motivated by the fact that the service differentiation at IP level is not sufficient, until packets with high priority can still be retarded by other packets from other classes at MAC layer. Where there must be a contention to access to the medium, which directly increase the average end-to-end delay .This proposition was maintained under an important number of assumptions: the adhoc network is connected to a fixed wired infrastructure, and the routing functionality is assured by a number of specific mobile devices. As in the case of cross-layers approaches, the results of this multi-layers combination were satisfactory for the various performance metrics: delay, jitter and loss rate. By so doing, we aim through this contribution, to extend this model to examine its efficiency in a distributed adhoc netwok architecture. 


\section{PROTOCOL ADAPTATION}

\subsection{Presentation of the adaptation process}

In spite of the multitude works about QOS in MANETS, there is no common process for treating similar problematic. Obviously, the design of a generic adaptation function will enable the determination of the starting point of all researches aiming to enhance previous works in this area. It is a necessity to define a global adaptation scheme, with the specification of the different parameters and variables involved, in order to help out understanding the whole adaptation work and also to be able to detect its faults and the possible improvements.

The adaptation in a communication system is defined as an alteration in the structure or the functioning of a protocol or any of its functions, resulting from the network environment variation, and by which the protocol will be able to perform better. Authors in [2] consider three sets of element in an adaptation process : network environment, which is perceptible through metric, behavior to adapt, the algorithms to be applied to depend on metric values, and performance to optimize. They define two forms of the adaptation process: Auto-Adaptation, and cross-layer adaptation. In both cases the process is considered dynamic, and is based on the metric calculation. However the adaptation process can be also static, in this case the auxiliary functioning is modified to meet certain requirements. We give the example of the EDCA, where the DCF algorithm is preserved but enhanced to support traffic differentiation. This can be considered as a static adaptation .In the dynamic case the adaptation process can be simplified in a mathematical order, it can be represented by a function $f(p)$, describing the process, while metrics are the variables deployed to adapt the protocol .Figure 1 resume the sets of elements acting in the adaptation of a giving protocol:

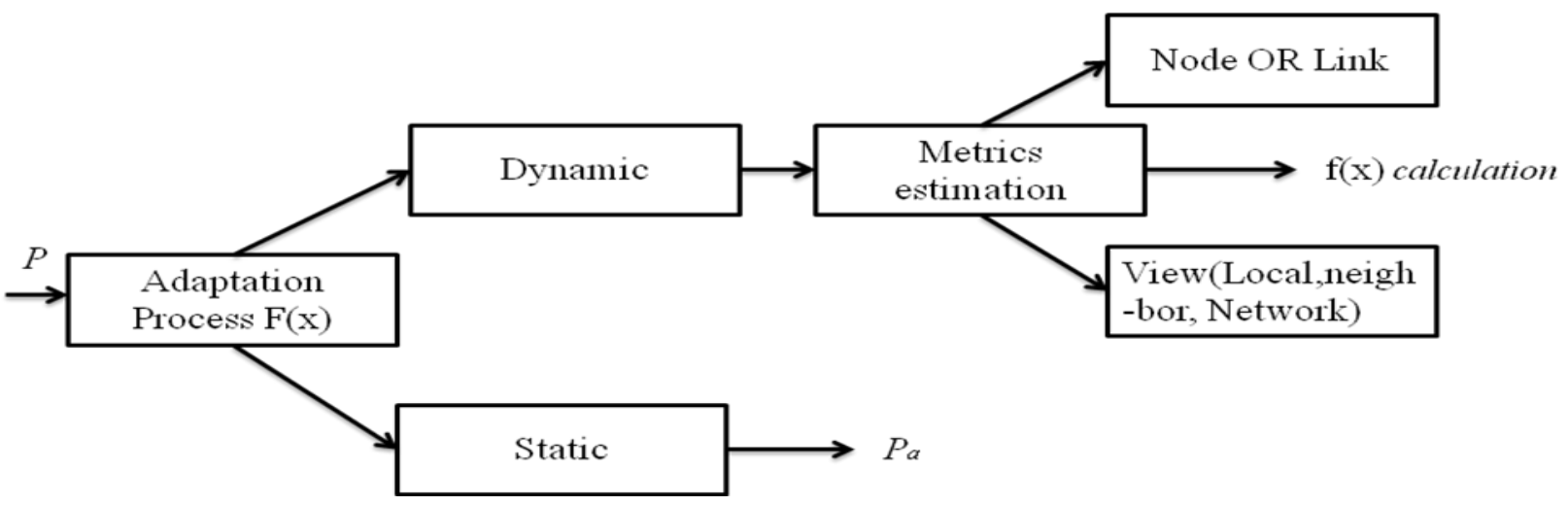

Fig 1: Elements of adaptation process

Let $g_{i}(p)$, be a specific property $\mathrm{f}$ a protocol $p, g_{i}$ can be a static parameter or a dynamic function with a number of variables, if we consider the example of the TCP, $g_{i}(p)$ could be the variation of the transmission or the congestion window. The adaptation process can be modeled by a mathematical function:

$$
\mathrm{f}\left(\mathrm{g}_{\mathrm{i}}(\mathrm{p})\right) \stackrel{\mathrm{x}_{1}, \mathrm{x}_{2}, \mathrm{x}_{3} \ldots \ldots \ldots \ldots \mathrm{x}_{\mathrm{n}}}{\longrightarrow} \mathrm{g}_{\mathrm{a}}(\mathrm{p})
$$

Where $x_{1}, x_{2}, x_{3} \ldots \ldots \ldots \ldots x_{n}$ represent the metrics used to adapt the protocol $p$ and $g_{a}(p)$ is the new adapted $g_{i}(p)$ according to the input metrics. In a linear program this formula can be reduced to an objective linear function (cost function) which depends directly on the network constraints:

$$
z=c_{1} x_{1}+c_{2} x_{2} \ldots \ldots \ldots \ldots c_{n} x_{n}
$$

This was a simple method for generalizing the adaptation process definition. The mathematical procedure can reduce

widely the complexity of the algorithm, but also it lights up the global parameters and the constraints being considered.

The complexity of the adaptation process depends mainly on the metrics estimation and calculation. If we consider the cross-layer adaptation approaches, we can notice that $90 \%$ of the information's need to be obtained from the physical and MAC (link) layers. However accurate results depend highly on realistic network conditions being simulated [3]. The assumption made about wireless channel and node physical characteristics must be defined at the beginning of the process. When we interest to the loss rate calculation, the environment nature must be considered, speaking about indoor or outdoor systems, and also the variations of the channel strength over time. Most works presented in this context relate, in a restricted way, for example, the loss rate calculation in wireless transmission to distance variation and propagation range value, which results a non-realistic formula, because of the impact of other multitude factors. Authors in [4] gave a deep review of the importance of the use of accurate wireless channel models for a good adaptation of protocol parameters. The section above gives an example of the complexity in some metrics estimation; we examine the end-to-end delay and bandwidth calculation.

\subsection{The complexity of metrics estimation:}

\subsubsection{End to End delay}

The delay is an additive metric. The average time to send a packet from a source to a destination node is equal to the sum of the average delays of the path components links. Delay estimation is essential in the context of designing a QoS routing protocol based on delay as metric.

The global delay is a composite of the following sub-delays:

-Transmission Delay: depends on the speed of the communication link. 
-Processing Delay :( also called latency: is the time for message processing a message. It depends on the capacity of processing node (power, CPU...) .

-Queuing Delay: depends on buffer size and the queuing politic and the transmission rate at node level and also on communication link.

-Propagation Delay: This delay depends on the physical characteristics of the communication link.

-Retransmission Delay: This delay depends on the error rate on the link and the protocol used for retransmissions.

In general the end-to-end delay is reduced to the following formula:

$$
D_{\text {global }}=D_{M Q D}+D_{M C D}+D_{M T D}[5]
$$

Where: $D_{M Q D}$ is the mean queuing delay, $D_{M C D}$ is the mean contention delay and $D_{M T D}$ :The mean transmission delay.

The delay calculation is related to a very complex theory. The required information's is highly probabilistic and relativist. It depends mainly on a multitude of parameters as: physical properties of the channel, the number of stations involved in the communication...., which are impossible to predict in most cases. Most works about optimization using the delay as metric, consider only the propagation delay, marginalizing the rest of the components, which affects, in reality, the credibility of the obtained results.

\subsubsection{Bandwidth:}

In MANETs, flows that traverse the same geographic neighborhood compete to have the best capacity in the wireless channel. Problems of collisions, interference and loss significantly slow their passages. Determining the capacity of an ad hoc network is a difficult problem since it depends on several parameters: the correlations between neighborhood links (the correlation is expressed in terms of interference that generates collisions, retransmissions, and even losses), the traffic model, the density of nodes, buffer size, mobility, etc. Authors In [6] developed a per-neighbor available bandwidth estimation scheme for IEEE 802.11-based wireless networks, in which they leverage the protocol's mechanisms to deal with contention and physical channel errors. Another method was proposed in [7] to evaluate the available bandwidth in adhoc networks based on the IEEE 802.11 MAC layer. This method combined the nodes' carrier sense capability to other techniques such as collision prediction to perform this estimation. Reference [8] proposes a new approach aiming to reveal the interference problem in the bandwidth estimation.

Whether is passive or active, the proposed solutions for the bandwidth estimation usually focused on evaluating the percentage of channel occupation by useful traffic, without considering the supplement part generated by interference, which leads to an approximate calculation, but remains far from the realistic value.

There is a large number of metrics used to enhance protocols performance. Those metrics are intended to manifest the real and implicit impact of a wireless transmission, or a certain physical propriety of a specific communication device. They can express the effect of one or more parameters with different correlation coefficients. The estimation and calculation of those parameters remain the most complicated step in adaptation process, especially with the high probabilistic nature of the wireless media.

\section{MIA \\ (MULTI-LAYER- INDEPENDENT -ADAPTATION) FOR MANETS}

Unlike Cross-layer adaptation, where protocols, operating at different layers from the protocol stack, exchange information's (directly or not), to adapt their functioning to a certain network constraint, multi-layer adaptation is a combination of a number of self-adaptation processes, triggered at different layers, but independently. As cited above, cross-layer adaptation has a several advantages, but the strong dependence between participant protocols limits choices about the communication protocols already used in a specific scheme. So in order to keep the validity of our approach although the modification of one contributor protocol, we choose to implement the quality of service at different layers. Each protocol will use its proper resources to respond the type of service required by an application. To achieve this objective, we opted for three quality of services mechanisms: QOS routing with an OLSR extension with QOS support, EDCAF (Enhanced Distributed Coordinate Function), and FEC (forward error correction). Our goal, through the combination of all those mechanisms is to offer a higher level of QOS, especially by enhancing the average end-to-end delay for delay sensitive traffics. QOLSR is a proactive routing protocol, and the type of traffic is not always known when routes calculation is performed. This fact leads us to say, that paths with QOS will convey both traffics with and without constraints, hence the idea of adding a priorities management at MAC level that will give a certain privilege to delay-sensitive applications. This distinction will fill the absence of a service differentiation at routing level on the one hand, and improve the local delay latency for realtime traffic on the other hand. EDCA aims then to add the differentiation of traffics in order to privilege those with high priority for the channel access, the same for the use of the FEC . They are both meant to enhance the end-to-end delay by avoiding the increase of the number of retransmissions. We prove that cooperating those three QOS mechanisms, even in a complete independent and primitive way enhances the network performance. Our study, aims to demonstrate the reliability of these assumption in the first place, and to locate its validation perimeter in a second place, especially for the real time traffics.

\subsection{QOLSR}

OLSR is a routing protocol [9] for mobile adhoc networks, it inherits the use of the link state algorithm, using shortest path algorithm to calculate routes, it sends periodic Hello messages locally to detect neighbor changes and TC messages to exchange topology information .OLSR uses a Multipooit technique to optimize diffusion, where only MPR nodes are responsible for forwarding control traffic. MPRs are alones used to form route from any source to any destination in the network.

OLSR has two principal functionalities: MPR selection and route calculation. For the MPR selection, the well known algorithm is the simple greedy [10], where MPR nodes are selected based on their coverage degree on two-hopneighbors. The RFC3626 proposes a new algorithm based on the introduction of a new parameter: the willingness, a value between 0 and 7 aiming to express the ability of a node to become an MPR. Theoretically this value is related to its residual energy, but none of the actual implementation of OLSR , is considering this factor into the MPR selection 
process. For the route computation, OLSR uses the dijkstra algorithm, with the constraint of the minimum hop number.

To make OLSR QOS aware, a number of approaches were proposed by the literature, some of them aim to modify the MPR selection algorithm, so that MPR nodes can by selected with the respect of a number of QOS constraints [11], other works choose to ensure QOS at routing level[12], with the use of a modified Dijkstra algorithm where the hop count is no longer the principal metric. A Number of propositions tried to enhance the two functions, in order to offer a high QOS level.

In this context,we evaluate the performance of three OLSR extensions : ETX_OLSR [13] which meant to find highthroughput paths on multi-hop wireless networks, ML_OLSR with the minimum loss as metric[14] for route calculation and MD_olsr [15],where the constraint considered is the minimum delay. In this paper we focus on enhancing the end-to-end delay for multimedia application, so we choose to deploy the extension with the minimum delay, in combination with the rest of chosen QOS mechanism.

\subsection{EDCAF}

EDCF is an extension of the access method DCF by the introduction of service differentiation. EDCF differentiates access to the medium using the principle of priorities. The DCF algorithm has not been changed completely in EDCF, but the game of time interval on which it is based has been customized for each priority. Accordingly these time intervals are adjusted to increase / decrease the probability of channel access and thus encourage / discourage the transmission of data high flow / low priority. We distinguish a total of eight levels of user priorities (UP or User Priority). Each priority is linked to what is called an access category (AC or Access Category). A CA, for its part, is characterized by its specific queue[16]. Each category is assigned an access logical function similar to DCF, called EDCAF (Enhanced Distributed Channel Access Function) which will participate in the contention phase in order to obtain a TXOP for the benefit of the concerned $\mathrm{AC}$ and then enable it to access the medium. The EDCAF works as DCF, following a CSMA/CA scheme with BEB, it is distinguished by the use of a lot of contention parameters of its own (the value of the AIFS and contention window interval [CWmin, CWmax] and a limited size of the TXOP). Although the distributed EDCA is an important enhancement to the original 802.11, it is not enough to provide QoS guarantees. The non-deterministic nature of the EDCAF, besides the absence of any distributed admission control algorithm, limit the enhancement provided by this access function, especially when the network conditions start to deteriorate (high number of station, mobility.....). Another problem is revealed about the static values of different EDCAF parameters (TXOP, Cwmin,Cwmax..), that remind the invariable, regardless the environment constraints or the traffic proprieties. So it appears that the EDCF parameters can require significant tuning to achieve performance goals. Several works were proposed to enhance the EDCA function, by optimizing the network performance via the fine-tuning of the EDCF parameters. Or optimizing the EDCF channel access by adapting the EDCF parameters including the TXOP limit during the run-time depending on the network load and supported applications. We cited as an example [17] where the high-priority traffic can reserve TXOPs by requesting admission for its traffic stream. The request is handled internally within the sender.

\subsection{FEC}

Forward error correction (FEC) consists on sending, in addition to the original information, redundant data, to enable a corrupted packet over the wireless link, to be reconstructed at the output of the link without the need of its retransmission. The use of FEC is suitable for real time applications. In wireless area The FEC can be used to avoid the frequent retransmission caused by the high packet loss rate at MAC level, to enhance the packet transmission delay, and decrease the number of drooped packet due to the exceed of retransmission threshold. The drawback of FEC is that it increases the network load and will then consume some additional bandwidth to transmit its redundant information. to resolve such constraint, authors in [18] present an hybrid model at the link-level combining FEC and ARQ-SR (Automatic Repeat Request with Selective Repeat), this combination aims to decrease the FEC needed for loss recovery. And reduce the impact of ARQ-SR on the RTT as the number of retransmission required to cover a lost frame is now smaller. Authors in [19][20] proposes an adaptative FEC , depending on the loss rate present in the network, another adaptative FEC technique was presented in [21], for a mobile wireless sensor network platform, called that adjusts the FEC code size based on the channel status indicated by acknowledgement packets' arrival. A recent work [22] presents a method that adapts dynamically the transmission rate and FEC, the aim goal of this approach was to maximize the transmission of the multicast video over a multi-rate wireless networks.

Technics proposed above, enhance network performances: packets losses , throughtputs (compared with the static FEC), and the recovery rate, but, under certain assumptions about the wireless channel model, defined by a number of physical parameters including propagation model, error model, channel state, path loss model.. etc. The difficulty to conceive a generic function for adaptative FEC is due principally to the complexity of wireless channel state prediction, because of its high probabilistic behavior. In our QOS model, we consider a simple model of an hybrid FEC /ARQ, we aim to avoid the deployment of the FEC when the channel present o low error rate .We use a simple function, based on measuring the packet losses rate in the network, will decide to add a redundant information's to packets (FEC) or not . When FEC fails to correct errors, the retransmission mechanism will be invoked. We define a static amount of redundancy, and consider an optimal value of the BER adapted to a typical indoor wireless environment. We modeled channel error by a bernouli process, where each value is generated statistically independent of previous output, The reason behind choosing such a simplest model, is that QOS is assured at other levels, and the usage of FEC here, has a further role in enhancing QOS metrics especially the end-to-end-delay.

\section{SIMULATIONS AND RESULTS}

We use network simulator ns2.34 [23], We simulate a network of 50 nodes with an area of $1000 * 1000$. We model a static adhoc network; under a TwoRayGround propagation model. We use $802.11 \mathrm{~b} / \mathrm{e}$ for MAC layer with a data rate of $11 \mathrm{Mb}$.We define two types of traffic: voice and best effort. The table above present different simulation parameters: 
Table1: Simulation parameters

\begin{tabular}{|l|l|l|}
\hline & voice & ftp \\
\hline Output(Mbits/s) & 0.064 & 1 \\
\hline $\begin{array}{l}\text { Packet } \\
\text { length(bytes) }\end{array}$ & 160 & 501 \\
\hline $\begin{array}{l}\text { Interarrival } \\
\text { time(s) }\end{array}$ & 0.02 & 0.025 \\
\hline Priority & 0 & 2 \\
\hline
\end{tabular}

We compare first the performance of the three OLSR extensions with QOS support. Figure 2 and 3, present respectively the E2Edelay and the percentage of loss rate for native OLSR with Hop count metric[24], ML_OLSR, MD_OLSR, and ETX_OLSR [25] in function of the ftp connection number.

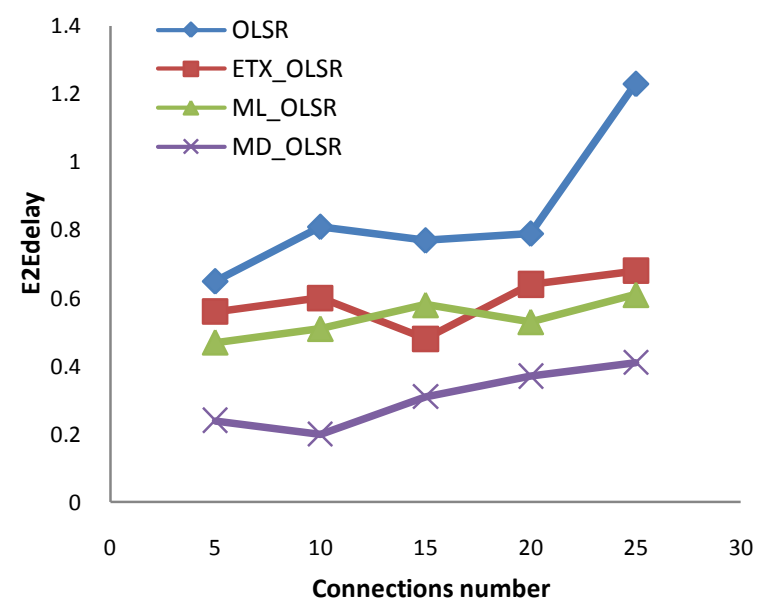

Fig 2: e2edelay for different OLSR extensions

Figure 2 demonstrates that MD_OLSR perform better For the e2edelay, due to the technique employed for the transmission delay calculation. Since OLSR-MD considers links with low delay using AdHoc Probe, a link capacity estimation technique. The higher delay value is obtained for native OLSR and the ETX_OLSR.

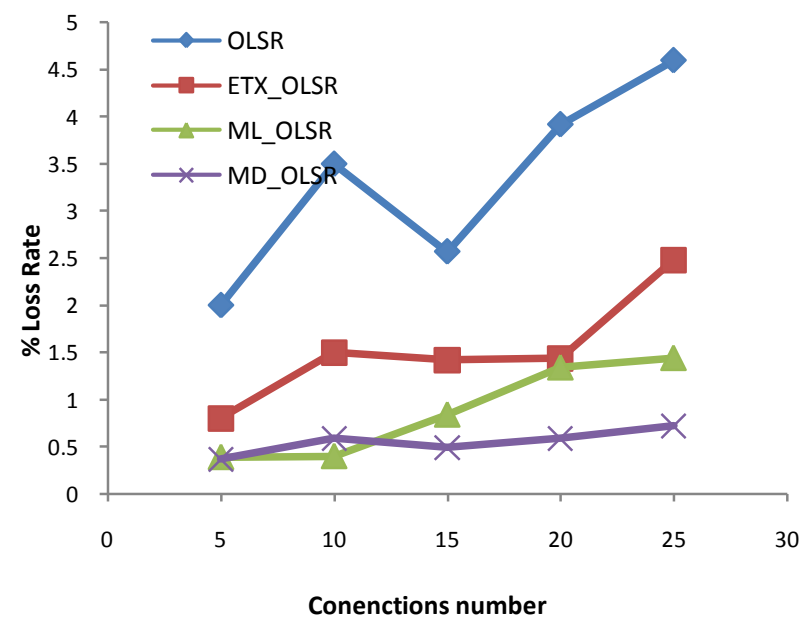

Fig 3:\% Loss Rate for different OLSR extensions
Comparing the loss rate for the four OLSR extensions, we can observe that ML_OLSR and MD_OLSR perform better. For the ML_OLSR, the use of the minimum loss as metric is meant to reduce the packet loss rate, by selecting routes with the minimum percentage of loss. As For the result obtained for the MD_OLSR, The explanation for this is that routes composed of shorter hops will be selected, since shorter hops have lower delays. This will allow reducing the probability of packet loss incidence, since routes are now composed of nodes that are closer to each other.

In our evaluation we choose to deploy the MD_OLSR extension, due to the optimal value obtained for both delay and loss rate, especially for the delay, which proved to be a relevant metric especially for application for which delay turn to be a crucial parameter.

We measure the end-to-end delay, the loss rate and the useful throughputs for MD_OLSR and MD_OLSR combined with EDCA , and MD_OLSR with the use of both EDCA and FEC.the graphs are given in Figure 4,5 and 6 accordingly.

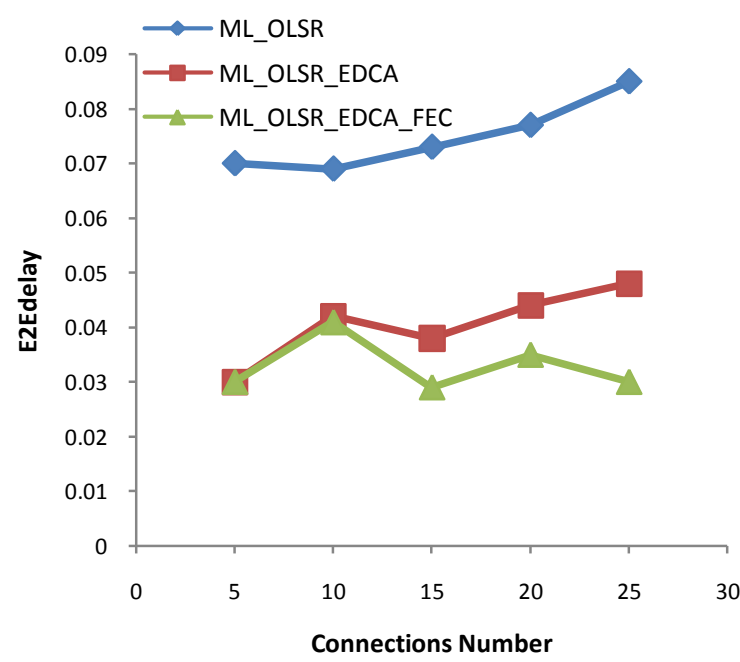

Fig 4:E2edelay for MD_OLSR, with EDCA and with EDCA/FEC

According to Figure 4, MD_OLSR combined with EDCA performs better than the OLSR alone, the contribution of the EDCA function alone is vastly observed with the decrease of the local delay for the voice application, to which is accorded the highest priority. As for the OLSR_EDCA and OLSR_EDCA_FEC, we can notice that for low traffic load (5-10 FTP connections), MD EDCA and MD_EDCA/FEC give almost the same result. This is can be explained by the fact that, the loss rate did not exceed yet the threshold defined for the use of FEC. With the increase of the number of FTP connections, congestions occurred, which means links will manifest a high loss rate, which will trigger the use of the FEC. This will avoid the frequent retransmissions, which has as a result, the decrease of the global delay. 


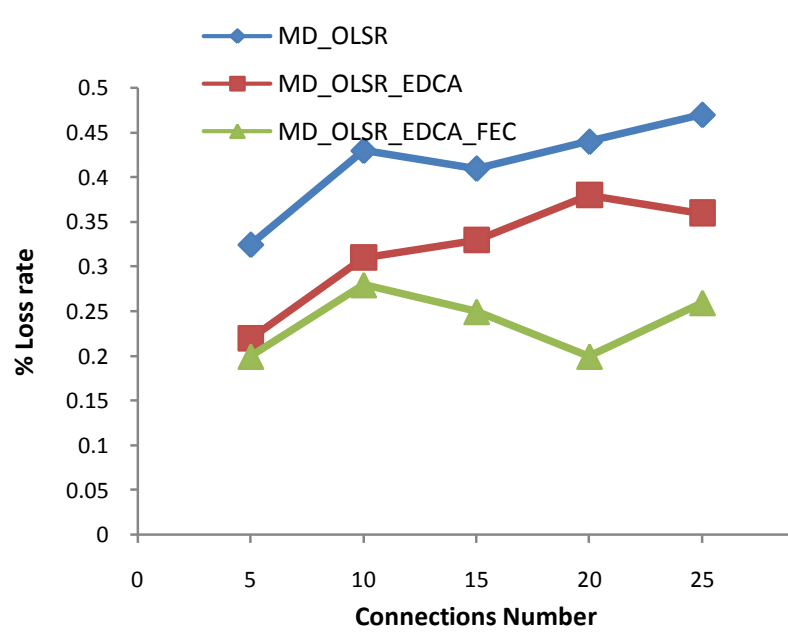

Fig 5:\% Loss Rate for MD_OLSR, with EDCA and with EDCA/FEC

As shown in Figure 5, the loss rate obtained with the use of the EDCA is lower than that obtained for the MD_OLSR alone. This can be explained by the fact that with the high priority accorded to the voice traffic, the highest value of the TXOP is then got, so the voice packets will occupy the channel without the need to content to access the channel each time for the defined TXOP interval. The use of FEC for error correction will reduce further the loss rate indicated by the reception of the NACK compared with OLSR with and without the EDCA. As shown the loss rate has significantly decreased with the use of the FEC.

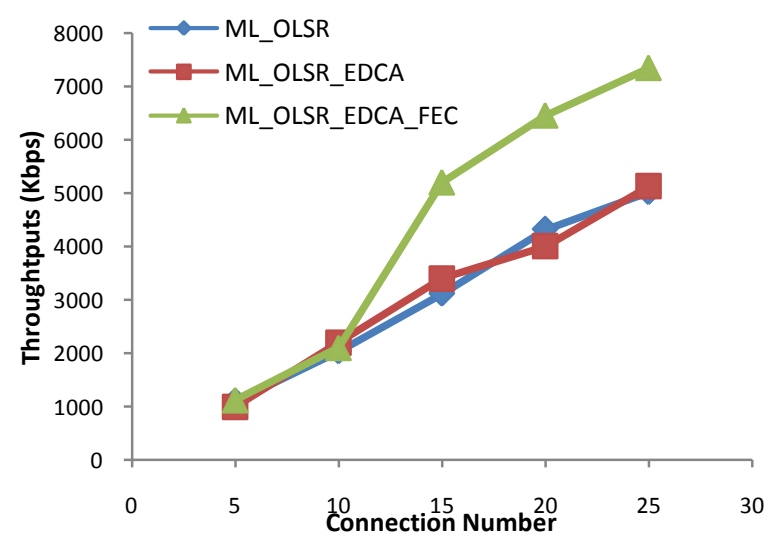

Fig 6: Throughtput(Kbytes/s) for MD_OLSR, with EDCA and with EDCA/FEC

Now consider Figure 6, here the use of the FEC will give the highest throughputs compared with MD_OLSR and MD_OLSR/EDCA. The FEC adds redundant information to packets to enable their correction at the receiver side. As we can see, the increase of the throughputs value did not start with the beginning of the transmission, but after detecting that the channel presents a loss rate exceeding the threshold fixed for the activation of the FEC function. Notice that we did not consider the retransmitted data, for the throughputs calculation.

\section{CONCLUSION}

We demonstrate through this paper that even the combination of three QOS mechanisms operating at different network layers, and working in a separate way, could be very useful . We concentrate in this paper on enhancing the end-to-end delay; MD_OLSR improves the transmission delay by selecting routes with minimum delay while EDCA enhance the local delay by privileging traffics with high priority. The use of FEC allows avoiding frequent retransmissions which has an impact on the global delay. Our goal was to prove the efficacy of the use of an hybrid multi-layers QOS scheme, that's combines static (EDCF) and dynamic adaptation (FEC \& OLSR), so we choose the standard model of the EDCA and a simple model of FEC/ARQ. This approach can be improved by adapting EDCA and FEC in a more exhaustive way to the network variations. Tuning EDCA parameters for example can enhance the result .We investigate currently the use of other metrics, bandwidth for example, in order to make protocols more aware about the network status.

\section{REFERNECES}

[1] R. Meraihi,G. Le Grand, S. Tohmé, M. Riguidel "Gestion multicouches de la qualité de service dans un réseau ad hoc à cœur stable " Proc. of CFIP 2003

[2] C. Yawut, B. Paillassa, R Dhaou ," Adaptation Process for Ad hoc Routing Protocol" In Proc, of the $10^{\text {th }}$ International Conference on Innovative Internet Community Services 2010, Thailand

[3] G A. Konrad, B. Y. Zhao, A. Joseph, and R. Ludwig. A "Markov-based channel model algorithm for wireless networks," In Proc. of ACM MSWiM, 2001.

[4] A. Konrad, B. Y. Zhao, A. Joseph,"Choosing an Accurate Network Model using Domain Analysis" Parallel and Distributed Systems, 2005. Proceedings. 11th International Conference.

[5] H. Shah, K. Chen, K. Nahrstedt "Available Bandwidth Estimation in IEEE 802.11-based Wireless Networks" Proc of 1st ISMA/CAIDA Workshop on Bandwidth Estimation 2003.

[6] C. Sarr, C. Chaudet, G. Chelius, I. Guérin Lassous "Bandwidth Estimation for IEEE 802.11-Based Ad Hoc Networks" Mobile Computing, IEEE Transactions 2008.

[7] D. Nguyen, P. Minet "QoS support and OLSR routing in a mobile ad hoc network" International Conference on Systems and International Conference on Mobile Communications and Learning Technologies, 2006.

[8] Jacquet, A. Laouiti, P. Minet, P. Muhlethaler, A. Qayyum, L. Viennot: Optimized Link "State Routing Protocol”, RFC 3626, IETF, 2003.

[9]A. Qayyum ,L. Viennot,A. Laouiti "Multipoint Relaying for Flooding Broadcast Messages in Mobile Wireless Networks",Aug 2002.

[10] Z. Li, N. Yu, Z. Deng" NFA: A new algorithm to select MPRs in OLSR"WiCOM 08.

[11]Badis,H.,Al Agha,K.,'Ddistributed algorithms for single and multiple metric link state qos routing " IFIP MWCN'03.

[12] D. S. J. De Couto, D. Aguayo, J Bicket , R. Morris,” A High-Throughput Path Metric for Multi-Hop Wireless Routing "Proc. Mobicom,2003. 
[13] Diego Passos , D.V Teixeira, C. Muchaluat-Saade , C. Schara Magalhães, V. N. Albuquerque," Mesh Network Performance Measurements “

[14] W. Cordeiro, E. Aguiar, W. Moreira Júnior, A. Abelém, M. Stanton," Providing Quality of Service for Mesh Networks Using Link Delay Measurements" Computer Communications and Networks, 2007.

[15] K. Kawamura, T. Hiraguri, M. Ogasawara," Technique for Dynamically Updating EDCA Access Parameters for WLANs" NTT Technical Review, Nov. 2007, Vol. 5, No. 11.

[16] S. Ahsanuzzaman, Md. Tariq , F. Granelli, ” Performance Analysis of Wireless Ad-Hoc Network Based on EDCA IEEE 802.11e" World Academy of Science, Engineering and Technology 69, 2010.

[17] A. Hamidian and U. Korner," An enhancement to the IEEE 802.11e EDCA providing QoS guarantees", Telecommunication Systems, Volume 31, March 2006

[18] A. Al Fawal ,C. Barakat "Simulation-Based Study Of Link-Level Hybrid FEC/ARQ-SR For Wireless Links and Long-Lived TCP traffic" WiOpt'03: Modeling and Optimization in Mobile, Ad Hoc and Wireless Networks, Mar. 2003.

[19] C. Padhye ,J. Christensen, W. Moreno" A New Adaptive FEC Loss Control Algorithm for Voice Over IP
Applications" Performance, Computing, and Communications Conference, 2000. IPCCC '00. Conference Proceeding of the IEEE International.

[20] Gandikota, V.R. Tamma, B.R. Murthy," Adaptive FECBased Packet Loss Resilience Scheme for Supporting Voice Communication over Ad hoc Wireless Networks”Mobile Computing, IEEE Transactions 2008.

[21] J. Ahn, S. Hong, J. Heidemann "An Adaptive FEC Code Control Algorithm for Mobile Wireless Sensor Networks" Journal of Communications and Networks 2005.

[22] Ö. Alay ,T. Korakis ,Y.Wang "Dynamic Rate and FEC Adaptation for Video Multicast in Multi-rate Wireless Networks" ;Mobile Netw Appl (2010).

Shivendra Panwar

[23] "The network simulator," http://www.isi.edu/nsnam/ns/, cited July 2011

[24] OLSR patch http://masimum.inf.um.es/fjrm/?page_id=116.

[25] OLSR modules for NS2 (OLSR-MD, OLSR-ETX, OLSR-ML OLSR), http://www.inf.ufrgs.br/ wlccordeiro/resources/olsr/. 